\title{
Wave transport and statistical properties of an open non-Hermitian quantum dot with parity- time symmetry
}

B Wahlstrand, Iryna Yakymenko and Karl-Fredrik Berggren

\section{Linköping University Post Print}

\section{Tweet}

N.B.: When citing this work, cite the original article.

Original Publication:

B Wahlstrand, Iryna Yakymenko and Karl-Fredrik Berggren, Wave transport and statistical properties of an open non-Hermitian quantum dot with parity-time symmetry, 2014, Physical Review E. Statistical, Nonlinear, and Soft Matter Physics, (89), 062910.

http://dx.doi.org/10.1103/PhysRevE.89.062910

Copyright: American Physical Society http://www.aps.org/

Postprint available at: Linköping University Electronic Press http://urn.kb.se/resolve?urn=urn:nbn:se:liu:diva-107613 


\title{
Wave transport and statistical properties of an open non-Hermitian quantum dot with parity-time symmetry
}

\author{
B. Wahlstrand, ${ }^{*}$ I. I. Yakimenko, and K.-F. Berggren ${ }^{\dagger}$ \\ Department of Physics, Chemistry and Biology, Linköping University, S-58183 Linköping, Sweden \\ (Received 14 October 2013; revised manuscript received 6 February 2014; published 10 June 2014)
}

\begin{abstract}
A basic quantum-mechanical model for wave functions and current flow in open quantum dots or billiards is investigated. The model involves non-Hertmitian quantum mechanics, parity-time $(\mathcal{P} \mathcal{T})$ symmetry, and $\mathcal{P} \mathcal{T}$-symmetry breaking. Attached leads are represented by positive and negative imaginary potentials. Thus probability densities, currents flows, etc., for open quantum dots or billiards may be simulated in this way by solving the Schrödinger equation with a complex potential. Here we consider a nominally open ballistic quantum dot emulated by a planar microwave billiard. Results for probability distributions for densities, currents (Poynting vector), and stress tensor components are presented and compared with predictions based on Gaussian random wave theory. The results are also discussed in view of the corresponding measurements for the analogous microwave cavity. The model is of conceptual as well as of practical and educational interest.
\end{abstract}

DOI: 10.1103/PhysRevE.89.062910

PACS number(s): 05.45.Mt, 03.65.-w, 73.63.-b

\section{INTRODUCTION}

In ballistic wave transport through various resonators connected to the surroundings via input and output leads or other probes, one frequently encounters scattering wave fields $\phi(\mathbf{r})$ with generic morphology and statistical behavior. Examples are open quantum dots or cavities, planar microwave billiards, acoustic reverberation rooms, optical cavities and microchip lasers, special electric $R L C$ networks, etc. [1-5]. Many times the analysis of the underlying dynamics of such systems may be reduced to Schrödinger or Helmholtz types of equations. In many cases the different systems may then be mapped formally onto each other. For example, two-dimensional nanosized quantum dots may be emulated by macroscopic planar microwave resonators [6]. Quantum wave functions, currents, etc., may thus be inferred from measurements on classical microwave analogs.

There is a great deal of interest in the modeling of systems as above. The main purpose of the present paper is to elaborate a basic heuristic model for electron states in open quantum dots (alternatively "cavities," "billiards," "corrals," "resonators," etc.). In a recent paper we have argued that quantum transport may be modeled by means of imaginary potentials to simulate the source and the sink for the carriers $[7,8]$. The model originates from early phenomenological inelastic nuclear scattering theory $[9,10]$ in which nuclear absorption is accounted for by a fictitious imaginary scattering potential. In our case, such a potential would act as a sink. This type of modeling was thus elaborated on in Refs. [7,8] by introducing an additional imaginary potential of opposite sign playing the role of a source. Having both the source and the sink embedded within the system, we may thus study the flow of particles from one lead to another while keeping the system formally closed. This is a neat simplification from a computational point of view. In addition, multileads, inelastic scattering events, etc., may be incorporated in the same simple fashion. At the same time

\footnotetext{
${ }^{*}$ Present address: HiQ Mälardalen AB, Arboga, Sweden.

${ }^{\dagger}$ Corresponding author: kfber@ifm.liu.se
}

there is the conceptual complication of non-Hermiticity. In this respect our model also offers obvious educational merits.

The method of imaginary potentials for source and drain as outlined in Refs. [7,8] has been applied to a "twolead" planar [two-dimensional (2D)] quantum dot in a gated $\mathrm{Al}_{x} \mathrm{Ga}_{(1-x)} \mathrm{As} / \mathrm{GaAs}$ heterostructure [11]. Particle densities and probability current flows for a selected set of low-energy states were compared with analog measurements for an equally shaped microwave resonator [12]. The resemblance between the two cases is noteworthy for the typical resonant states selected in that study.

The aim of the present work is to extend this kind of analysis to another class of states, namely, high-frequency chaotic states in an open billiard. We will thus ask the question whether the present approach will yield, for example, the well-established universal statistics for probability density and current distributions. Since we make use of non-Hermitian Hamiltonians (NHHs), results of this kind are of principle interest and are not obvious beforehand. In the same way we will also discuss the statistical distribution for the somewhat esoteric Pauli quantum-mechanical stress tensor from 1933 [13-15]. Usually the Pauli stress tensor makes only a small contribution to the total stress. Here we are fortunate to have a system in which the Pauli stress tensor is singled out and may be studied in detail.

As already indicated, the present model falls within the general framework of non-Hermitian quantum mechanics (NHQM) [16-19], which is gaining growing attention in a number of fields. Here we may mention especially inelastic quantum scattering and transport by complex potentials $V$ [20-26] and optical waveguides [27-29] that are all examples of applied NHQM. Eigenvalues of a non-Hermitian Hamiltonian are generally complex. However, of special concern here is that they may turn out to be real under certain conditions [16-19]. A necessary prerequisite is then that we deal with parity-time $(\mathcal{P} \mathcal{T})$ invariance [parity $\hat{p} \rightarrow-\hat{p}, \hat{x} \rightarrow-\hat{x}$ and time operations $\hat{p} \rightarrow-\hat{p}, \hat{x} \rightarrow \hat{x}, i \rightarrow-i$, respectively) As we will see, we must also have $V(\hat{x})=V^{*}(-\hat{x})$. In other words, $\mathcal{P} \mathcal{T}$ symmetry requires that the real part of the potential $V$ is an even function of position $\hat{x}$. Examples of $\mathcal{P} \mathcal{T}$ quantum 
mechanics are found in Refs. [27-29] for optical wires. It seems, however, that $\mathcal{P} \mathcal{T}$ invariance has received less or no attention in conventional condensed matter physics and nanodevices as here.

The outline of the article is as follows. In Sec. II we summarize quantum mechanics with complex potentials and at the same time put our model into the general framework of $\mathcal{P} \mathcal{T}$ invariance. In the same section we also discuss how a particular device should be designed to ensure $\mathcal{P} \mathcal{T}$ symmetry and thereby real eigenvalues and ballistic transport. Section III presents an application to a planar microwave resonator, an analog system to a quantum two-dimensional quantum dot. Computational methods and the nature of the $\mathcal{P} \mathcal{T}$-symmetric numerical solutions are also presented in the same section. To get more insight into the nature of these solutions, we discuss briefly a simple but useful approach based on standard perturbation theory as it illustrates $\mathcal{P} \mathcal{T}$ symmetry in a simple and transparent way. The interaction of close levels is also considered as it demonstrates $\mathcal{P} \mathcal{T}$-symmetry breaking. Statistical properties and numerics for density, current, and stress-tensor distributions are discussed in Sec. IV, together with brief comments on recent measurements and theory [30]. A discrepancy between theory and measurements observed previously for the stress tensor is shown to disappear with increasing frequency. The stress tensor, which until Ref. [30] has passed unnoticed in the context of wave function statistics, is of fundamental interest as it provides a delicate test of theory with its direct dependence on second-order derivatives of a wave function. A brief summary and concluding remarks are given in Sec. $\mathrm{V}$.

\section{WORKING OF IMAGINARY POTENTIALS}

\section{A. Conventional QM}

Fictitious imaginary potentials to mimic quantum transport and flow in an open ballistic quantum dot were described in Ref. [7]. For convenience, here we summarize the key steps starting from conventional QM. The basic ingredients are as follows: the time-dependent Schrödinger equation,

$$
i \hbar \partial \Phi / \partial t=-\frac{\hbar^{2}}{2 m} \Delta \Phi+V \Phi,
$$

where $V$ is the potential which is real and $m$ is the mass of the particle; the probability current density.

$$
\mathbf{J}=\frac{\hbar}{2 m i}\left[\Phi^{*} \nabla \Phi-\left(\nabla \Phi^{*}\right) \Phi\right]
$$

and the continuity equation,

$$
\partial \varrho / \partial t+\nabla \cdot \mathbf{J}=0
$$

where $\varrho=|\Phi|^{2}$ is the probability density.

The Pauli quantum-mechanical stress tensor (QST) is defined as [13-15] (see also Ref. [31])

$$
\begin{aligned}
T_{\alpha, \beta}= & \frac{\hbar^{2}}{4 m}\left(-\Phi^{*} \frac{\partial^{2} \Phi}{\partial x_{\alpha} \partial x_{\beta}}-\Phi \frac{\partial^{2} \Phi^{*}}{\partial x_{\alpha} \partial x_{\beta}}\right. \\
& \left.+\frac{\partial \Phi}{\partial x_{\alpha}} \frac{\partial \Phi^{*}}{\partial x_{\beta}}+\frac{\partial \Phi^{*}}{\partial x_{\alpha}} \frac{\partial \Phi}{\partial x_{\beta}}\right) .
\end{aligned}
$$

It features naturally in (semiconductor) quantum hydrodynamics [15] in which one deals with a "probability fluid." Flow lines and vorticity patterns are in that case closely related to QST.

If one restricts oneself to stationary states, the Schrödinger equation in (1) turns into its time-independent form

$$
-\frac{\hbar^{2}}{2 m} \Delta \phi(\mathbf{r})+V(\mathbf{r}) \phi(\mathbf{r})=E \phi(\mathbf{r}),
$$

where $E$ is the energy of the particle. For clarity we introduce the notations $\Phi(\mathbf{r}, t) \rightarrow \phi(\mathbf{r}) \exp (-i E t / \hbar), \mathbf{J}(\mathbf{r}, t) \rightarrow \mathbf{j}(\mathbf{r})$, and $\varrho(\mathbf{r}, t) \rightarrow \rho(\mathbf{r})$.

\section{B. Role of imaginary potentials}

According to (3) there is a conservation relation between $\varrho(\mathbf{r}, t)$ and $\mathbf{J}(\mathbf{r}, t)$ that holds for real potentials $V$. The idea of a potential containing also an imaginary component was, as mentioned, introduced long ago as an heuristic way of describing absorption in nuclear scattering $[9,10]$, i.e., in situations when particle conservation is not mandatory, in contrast to the present case of ballistic transport.

Assume that the complex $V$ is generally

$$
V=V_{R}+i V_{I},
$$

where $V_{R}$ and $i V_{I}$ are the real and imaginary parts, respectively. The generalized version of the continuity relation in (3) is then [10]

$$
\partial \varrho(\mathbf{r}, t) / \partial t+\nabla \cdot \mathbf{J}(\mathbf{r}, t)=\frac{2 V_{I}}{\hbar} \varrho(\mathbf{r}, t) .
$$

Since $\varrho(\mathbf{r}, t)$ is positive, the term on the right-hand side acts as a source or sink for positive or negative values of $V_{I}$, respectively. To make this more transparent one may integrate (7) over a volume $\Omega$ bounded by the surface $A$. Following [10] we then have

$$
\frac{\partial}{\partial t} \int_{\Omega} \varrho(\mathbf{r}, t) d \mathbf{r}=-\int_{A} \mathbf{J}(\mathbf{r}, t) \cdot \mathbf{n} d A+\frac{2}{\hbar} \int_{\Omega} V_{I} \varrho(\mathbf{r}, t) d \mathbf{r},
$$

where $\mathbf{n}$ is the unit vector normal to the surface. If the system is well contained in $\Omega$, the surface term vanishes, implying that the normalization integral decreases with time if $V_{I}$ is negative, i.e., particles are being absorbed. For positive $V_{I}$, on the other hand, the normalization increases as particles are created in this case.

As in Ref. [7] here we combine the two processes to study probability current flow (wave transport), vortices, etc., by giving $V_{I}$ different signs in different parts of the system. For example, to simulate the probability current density flowing between input and output leads in a cavity, we may simply replace the extended leads by two regions in which $V_{I}$ is nonzero and of opposite signs. The condition for a steady, well-balanced, and lossless flow between the two regions is

$$
\left\langle V_{I}\right\rangle=\int_{\Omega} V_{I} \varrho(\mathbf{r}, t) d \mathbf{r}=0 .
$$

Therefore the expectation value of the Hamiltonian operator $\langle H\rangle$ is real despite $V=V_{R}+i V_{I}$ being non-Hermitian. Furthermore, the corresponding eigenvalues are real when $V_{R}$ is independent of time. As we will see in the following sections, our elementary approach of using $\pm i V_{I}$ for a source and 
sink to emulate the probability current densities and transport properties of a cavity works quite well in such cases (in general also for other types of probes). With a current thus flowing from a source to sink, we refer to the cavity as an open one $[7,19]$. The situation is thus akin to the current flowing between the poles in an ordinary battery. As we will see below, however, there may also be closed vortical currents that do not contribute to the net flow through the cavity.

\section{Conservation of probability density}

The continuity equation (8) requires that $\left\langle V_{I}\right\rangle=0$ for states with stationary probability densities and currents. The problem with this condition is that $\phi(\mathbf{r})$ must be known before choosing $V_{I}$. Also, even if this condition holds for one state, it is unlikely to do so for the other ones that generally would turn out to have complex eigenvalues. So, in general, $V_{I}$ needs to be chosen with respect to a specific state that is to be studied. One may view this as a "selective filtering" of a particular mode. However, the system is not generally $\mathcal{P} \mathcal{T}$ symmetric in a situation as this. One should therefore incorporate $\mathcal{P} \mathcal{T}$ symmetry already in the design of a particular device in the following way. For geometrically symmetric confinements with $V(\hat{x})=V^{*}(-\hat{x}), \mathcal{P} \mathcal{T}$ symmetry and condition (9) are easy to meet, at least if $\left|V_{I}(\mathbf{r})\right|$ is chosen to be sufficiently small and the levels are well separated (cf. Sec. III C). If so, the states in such systems are in practice either symmetric or antisymmetric and the probability density is consequently symmetric. Thus, by choosing an antisymmetric imaginary potential, one satisfies the above conditions.

In the following we will focus on geometrically symmetric systems $\left[V_{R}(x)=V_{R}(-x)\right]$ which makes the following simulations more easy to handle. As we will see, there are some interesting features associated with the complex level interactions of odd and even states.

\section{APPLICATIONS TO A PLANAR NOMINALLY SYMMETRIC MICROWAVE RESONATOR}

As indicated in the Introduction, quantum systems may be emulated many times by classical analogs. Well-known examples are various flat microwave resonators for which there is a one-to-one correspondence between the Helmholtz equation and the stationary Schrödinger equation, including the Dirichlet boundary conditions [2,6], i.e.,

$$
\left(\Delta+k^{2}\right) \phi(\mathbf{r})=0 .
$$

Here $\phi(\mathbf{r})$ represents the perpendicular electric field $E_{z}(x, y)$ that mimics the quantum state; the QM wave number $k=$ $\sqrt{2 m E} / \hbar$ is replaced here by $k=\omega / c$, where $\omega$ is the angular frequency of the transverse magnetic (TM) mode and $c$ is the speed of light. The Poynting vector is the analog to the probability current density (in the following denoted by $\mathbf{j}$ as in QM). Experimental studies of generic phenomena such as wave morphology, flow patterns, etc., are thus possible.

In the following we focus on the cavity shown in Fig. 1, which is designed after a cavity used in a number of systematic microwave experiments [6,12], which in turn have been inspired by measurements of conductance and wave function scarring in source-drain biased open quantum dots with very

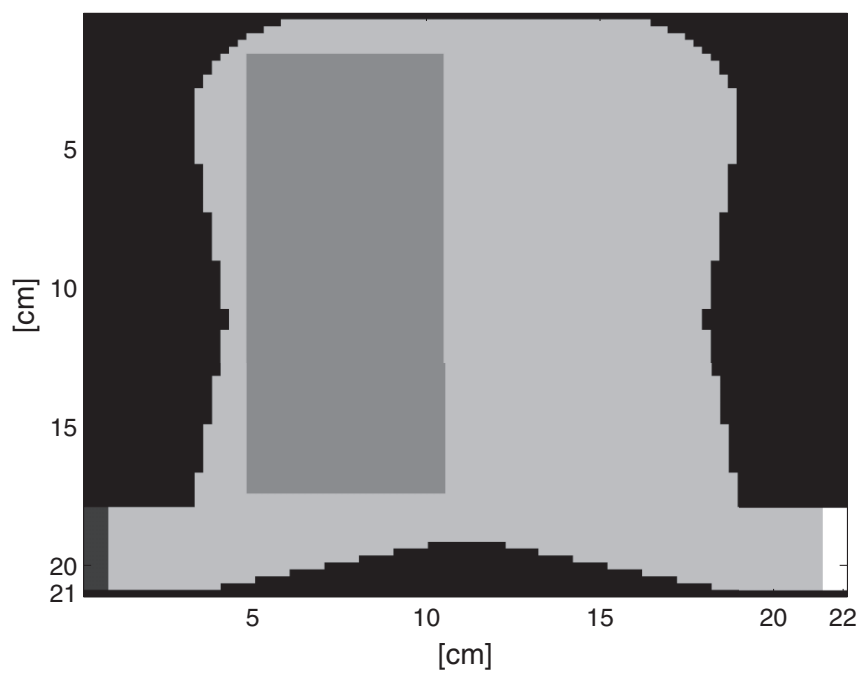

FIG. 1. A nominally symmetric hard-wall 2D cavity of height $21 \mathrm{~cm}$ and width $22 \mathrm{~cm}$ used in the calculations. The shaded rectangle is the region used to sample data for the statistical analysis. The cavity is shaped after a particular quantum dot [11]; at the bottoms of the two "quasileads" there are regions in which $V_{I}$ is positive or negative (dark and white regions, respectively). The steps along the boundaries indicate the rectangular computational grid.

much the same geometric form [11]. In our case we must, however, supplement the above equation with a complex term as

$$
\left(\Delta+k^{2}+i V_{I}\right) \phi(\mathbf{r})=0,
$$

with $V_{I}(x)=-V_{I}(-x)$. Except for the small source and drain regions in Fig. 1, the two equations are identical.

\section{A. Computational method}

To solve the 2D Eq. (11) one must turn to numerical methods because of the irregular form of the cavity in Fig. 1. We have chosen the finite difference method (FDM) [32] in which the $(x, y)$ space is discretized into a lattice $\left(x_{i}, y_{j}\right)$, with each point representing an eigenfunction $\phi\left(x_{i}, y_{j}\right)$. Hence (11) is turned into a (complex) matrix equation which may be solved with standard computer software.

The points $\left(x_{i}, y_{j}\right)$ are chosen to span a rectangular grid which is convenient from a computational point of view. More important, however, is that we may stay close to experiments [30]. In these experiments, the sample points were placed as a rectangular grid, just as the grid points in FDM. The number of points were chosen to ensure sufficient numerical accuracy. The numerical $\mathcal{P} \mathcal{T}$-invariant solutions are used in the following sections to evaluate the various statistical properties for densities, the Poynting vector (current), and the stress tensor distributions.

\section{B. Numerical solutions for $\phi\left(x_{i}, y_{j}\right)$ : General features}

Computed eigenvalues all turn out to be real except in the regions of close level interaction as in Sec. III D. Below we will present the typical behavior of the $\mathcal{P} \mathcal{T}$-symmetric solutions in two different frequency regimes. In addition, we will consider the case when two states are very close in energy and the reordering that may take place as the strength of $V_{I}$ is varied. 

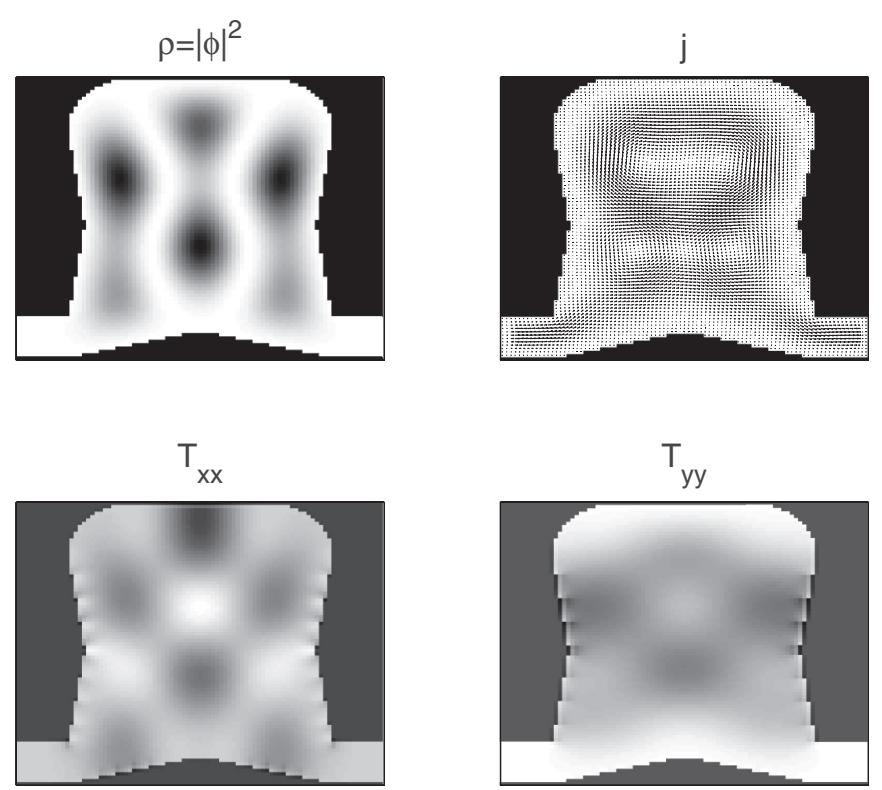

FIG. 2. Plots for frequency $3.09 \mathrm{GHz}$. Top left: $|\phi|^{2}$. Top right: The current $\mathbf{j}$. Bottom left: The stress tensor component $T_{x x}$. Bottom right: The stress tensor component $T_{y y}$.

Figures 2 and 3 show the computed densities $\left|\phi\left(x_{i}, y_{j}\right)\right|^{2}$, currents $\mathbf{j}$, and stress tensor components $T_{\alpha \beta}$ in low- and high-frequency regimes. In the first case the results are clearly structured with a simple "particle-in-a-box" type of behavior. In the second case the wavelength is much shorter than the dimensions of the enclosure. Hence the results are more fragmented this time.

\section{Perturbation method for small $\left|V_{I}\right|$}

To gain deeper insight into the nature of the solutions, one may turn to conventional perturbation theory. If $E_{n}^{0}$ and $\phi_{n}^{0}$
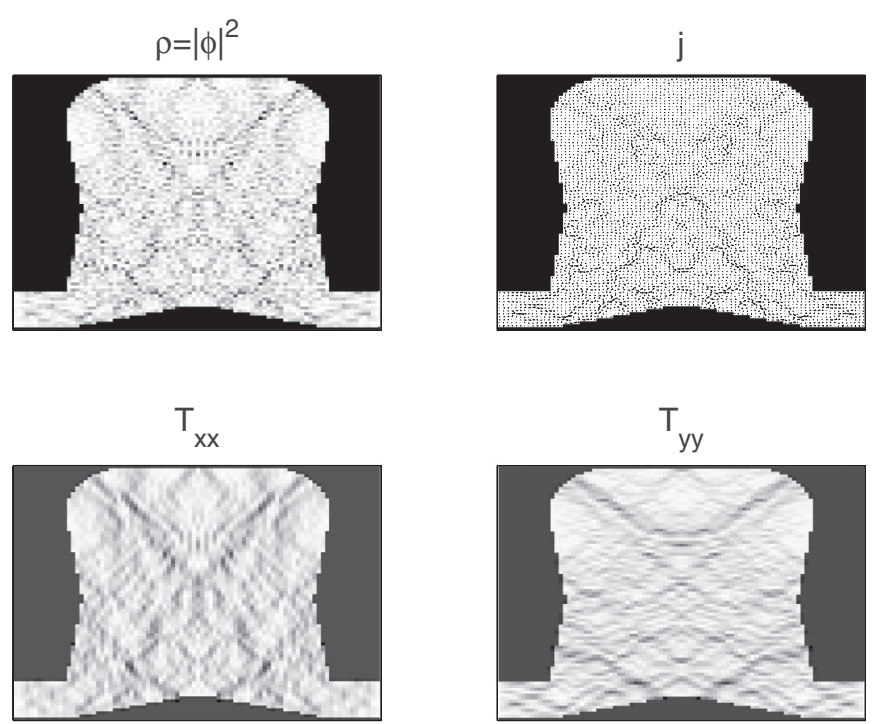

FIG. 3. Plots for frequency $31.98 \mathrm{GHz}$. Top left: $|\phi|^{2}$. Top right: The current $\mathbf{j}$. Bottom left: The stress tensor component $T_{x x}$. Bottom right: The stress tensor component $T_{y y}$. refer to the case $V_{I}=0$, the lowest-order correction to the unperturbed wave function is

$$
\phi_{n}=\phi_{n}^{0}+i \delta \phi_{n},
$$

with

$$
\delta \phi_{n}=\sum_{m, m \neq n} \frac{\left\langle m\left|V_{I}\right| n\right\rangle}{E_{n}^{0}-E_{m}^{0}} \phi_{m}^{0} .
$$

This means that the change of wave function with the onset of a finite $V_{I}$ is purely imaginary. In our case $V_{I}$ is odd (cf. Fig. 1). Hence symmetric states will only combine with antisymmetric ones and vice versa in the perturbation expansion (13). The states (12) therefore illustrate $\pm 1 \mathcal{P} \mathcal{T}$ invariance in a simple and transparent way. This kind of basic approach also turns out to be a practical calculational tool when levels are clearly separated and $V_{I}$ is small.

\section{Complex interaction of levels and $\mathcal{P} \mathcal{T}$-symmetry breaking}

There are some salient features when a pair of even and odd states are close in eigenfrequencies. At $V_{I}=0$ the states are obviously real. As $V_{I}$ is made finite, however, the two states will, as just mentioned, acquire imaginary components by a mixing among the unperturbed states. Following degenerate perturbation theory the level shifts are, however, real as long as $\left|V_{I}\right|$ is sufficiently small.

Figure 4 shows $\left|\phi\left(x_{i}, y_{j}\right)\right|$ (absolute values for clarity) for two states with close frequencies. Obviously there is a simple mix of states that is very similar to the unperturbed pair $\phi_{28}^{0}$ and $\phi_{29}^{0}$. Here the value of $\left|V_{I}\right|=0.05 \pi^{2} \mathrm{~cm}^{-2}$ is relatively small. As a consequence, the imaginary parts are much less than the real ones.

As $\left|V_{I}\right|$ is gradually increased, a complex reordering of the two states occurs. In this region the real and imaginary components of the wave functions have similar weights and the mixing is nearly symmetric. Eigenvalues are then complex,
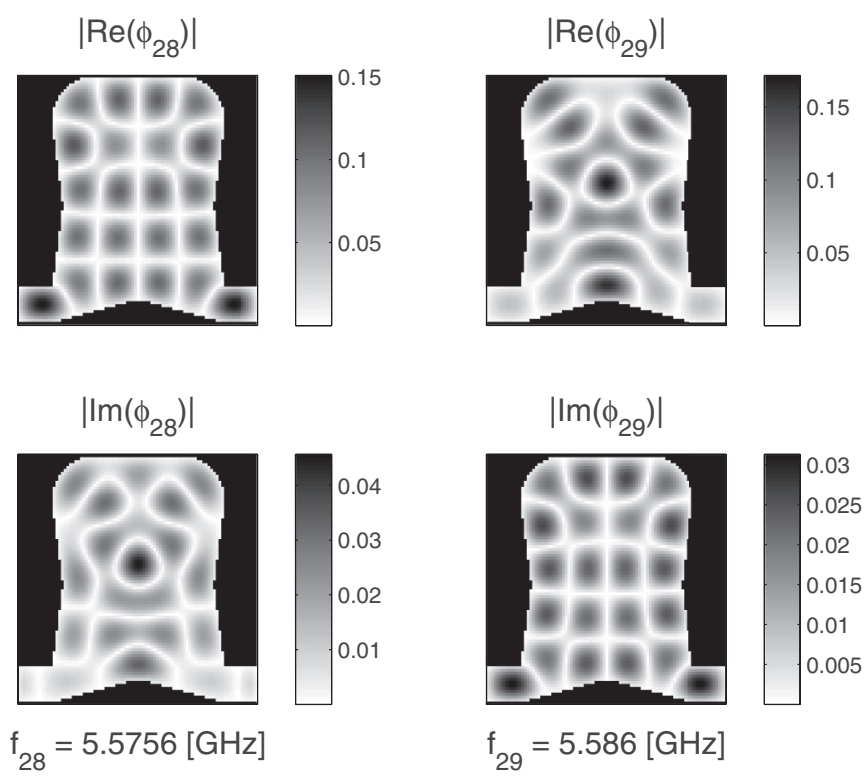

FIG. 4. Real and imaginary components of wave functions $\phi_{28}$ and $\phi_{29}$ for $\left|V_{I}\right|=0.05 \pi^{2} \mathrm{~cm}^{-2}$. 

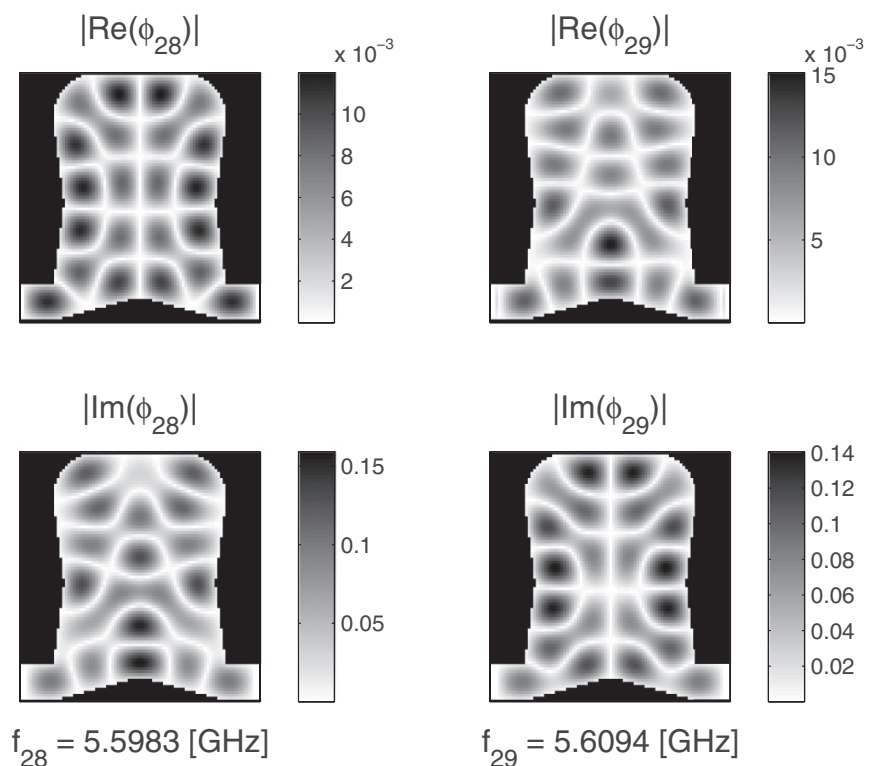

$\mathrm{f}_{28}=5.5983[\mathrm{GHz}]$

FIG. 5. Real and imaginary components of wave functions $\phi_{28}$ and $\phi_{29}$ for $\left|V_{I}\right|=10 \pi^{2} \mathrm{~cm}^{-2}$.

i.e., there is a complex level interaction and $\mathcal{P} \mathcal{T}$ symmetry is broken at the same time.

On further increase of $\left|V_{I}\right|$ beyond the region for reordering and complex level interaction the levels once more become real, but the two wave functions are now almost purely imaginary, as seen in Fig. 5, i.e., there is a phase rotation.

One component of the complex wave functions in Figs. 4 and 5 is thus dominant. In the region of level reordering, however, the real and imaginary components of the wave functions have, as mentioned, similar weights. This means that $\phi_{28}$ and $\phi_{29}$ become almost linearly dependent differing only by a phase factor, i.e., $\phi_{29} \approx i \phi_{28}$. At this point the eigenvalues become complex and the states become nonstationary. Related issues on wave function mixing and crossover of complex levels are found, for example, in Refs. [33-36].

\section{STATISTICAL DISTRIBUTIONS}

Chaotic quantum systems (and their analogs) have been shown to follow generic statistical distributions regarding density [2,37] (area scaled as $\rho=A|\phi|^{2}$ ), currents $\left(j, j_{\alpha}\right)$ [37], and stress tensor components $\left(T_{x x}\right.$ and $\left.T_{x y}\right)$ [30]. These distributions relate to Gaussian random waves and may be derived from the assumption that in each point the wave function can be described as a random superposition of monochromatic plane waves, as long as the point is sufficiently far from the boundary.

As a measure of openness of a cavity, one introduces a mixing parameter $\epsilon$ that ranges from 0 to 1 . When $\epsilon \rightarrow 1$ the cavity becomes fully open, and $\epsilon \rightarrow 0$, on the other hand, means that it is closed. This parameter is determined as [37]

$$
\epsilon=\sqrt{\frac{\left\langle q^{2}\right\rangle}{\left\langle p^{2}\right\rangle}}
$$

Here, the wave function

$$
\phi(\mathbf{r})=p(\mathbf{r})+i q(\mathbf{r})
$$

has been phase rotated so that $q$ and $p$ are statistically uncorrelated, i.e., $\langle p q\rangle=0$, and also $0 \leqslant \epsilon \leqslant 1$ [37]. Here we will focus on the cavity shown in Fig. 1. The dents on the sides of the cavity are introduced to suppress bouncing modes, which could bring elements of order to the system. The following distributions are obtained within conventional HQM assuming Gaussian random fields [37].

\section{A. Probability density distributions}

The statistical distribution of the density $\rho=A|\phi|^{2}$, here rescaled with respect to the area $A$ of the cavity, is [37] (see also Refs. [2,38])

$$
P(\rho, \epsilon)=\mu e^{\left(-\mu^{2} \rho\right)} I_{0}(\mu \nu \rho),
$$

where $I_{0}$ is the modified Bessel function of zeroth order and

$$
\mu=\frac{1}{2}\left(\frac{1}{\epsilon}+\epsilon\right), \quad v=\frac{1}{2}\left(\frac{1}{\epsilon}-\epsilon\right) .
$$

In the limit $\epsilon \rightarrow 0$ one recovers the Porter-Thomas distribution

$$
P(\rho)=\frac{1}{\sqrt{2 \pi \rho}} e^{-\rho / 2},
$$

which is the probability density distribution for wave functions that are invariant under time reversal and may be chosen to be real [37].

In the other limit $\epsilon \rightarrow 1$, the state is evidently complex with broken time symmetry and (16) then turns into the Rayleigh distribution [38]

$$
P(\rho)=e^{-\rho} .
$$

As seen in (18), the Porter-Thomas distribution is singular in $\rho=0$, while the Rayleigh distribution remains finite. In the real case, $|\phi|^{2}=p^{2}$, thus only $p$ needs be zero to have a zero density. This occurs along nodal lines. In the complex case, on the other hand, $|\phi|^{2}=p^{2}+q^{2}$. Now both the independent functions $p$ and $q$ must both vanish for $|\phi|^{2}$ to be zero. This happens only when the intersection points between the nodal lines belonging to $p$ and $q$, respectively. For a completely chaotic case both $p^{2}$ and $q^{2}$ obey the Porter-Thomas distribution (if properly rescaled with respect to A) and, when added together, their sum $|\phi|^{2}=p^{2}+q^{2}$ follows the Rayleigh distribution.

\section{B. Numerical probability density distributions}

Numerically obtained density distributions are shown in Figs. 6 and 7; guided by measurements, 1472 interior grid points (see Fig. 1) were normally used. However, at frequencies higher than utilized experimentally (5.5-10 GHz [30]) an increased resolution was required to bring simulations and the present random wave theory into quantitative agreement. The graphs in Figs. 6 and 7 clearly show the crossover from a practically closed system that obeys the Porter-Thomas distribution to an open system with a Rayleigh distribution. 


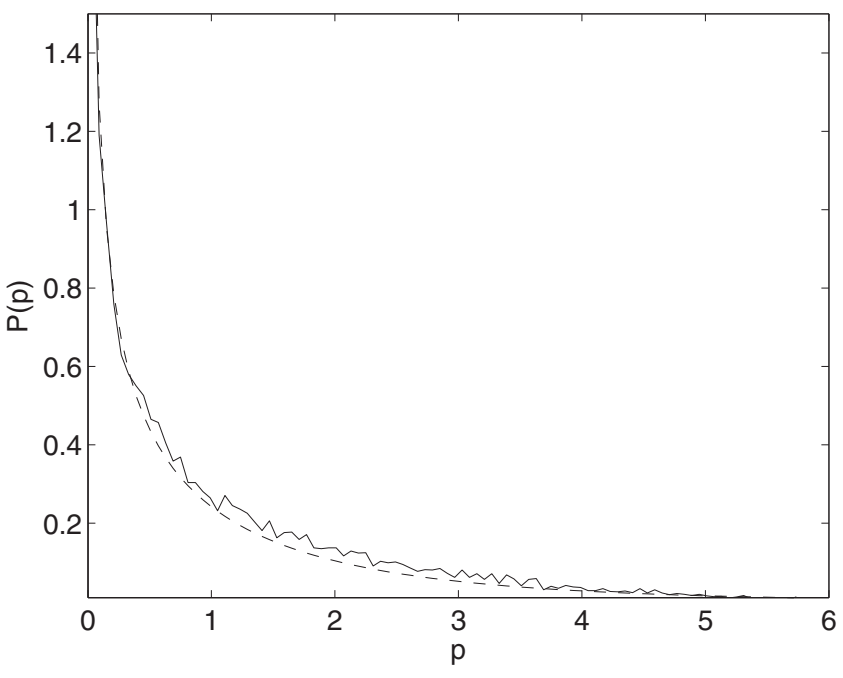

FIG. 6. Numerical probability density distribution (solid line) obtained as an average over 11 wave functions with frequencies between 4.8 and $5.92 \mathrm{GHz}$ and the predictions from the Gaussian random wave theory (dashed curve) in $(16) ;\langle\epsilon\rangle=0.124$.

As mentioned above, not all points are included to avoid possible effects of boundary symmetry. We thus find a slight dependence on frequency among the states being sampled, but the overall results are quite satisfactory. We do indeed find that NHQM with fictitious imaginary probes works in practice for the present $\mathcal{P} \mathcal{T}$-symmetric system. At the relatively high frequencies studied the method yields results in good agreement with predictions from conventional HQM random wave theory and statistics collected for realistic cavities. There is a slight dependence on frequency, but the overall agreement is nevertheless good.

At lower frequencies, on the other hand, one finds that fluctuations increase in magnitude and as a consequence there are deviations from the ideal distribution in (16). This happens because the wavelength increases in this case to the extent that boundary effects become noticeable as in Fig. 2. This general behavior is expected and well known.

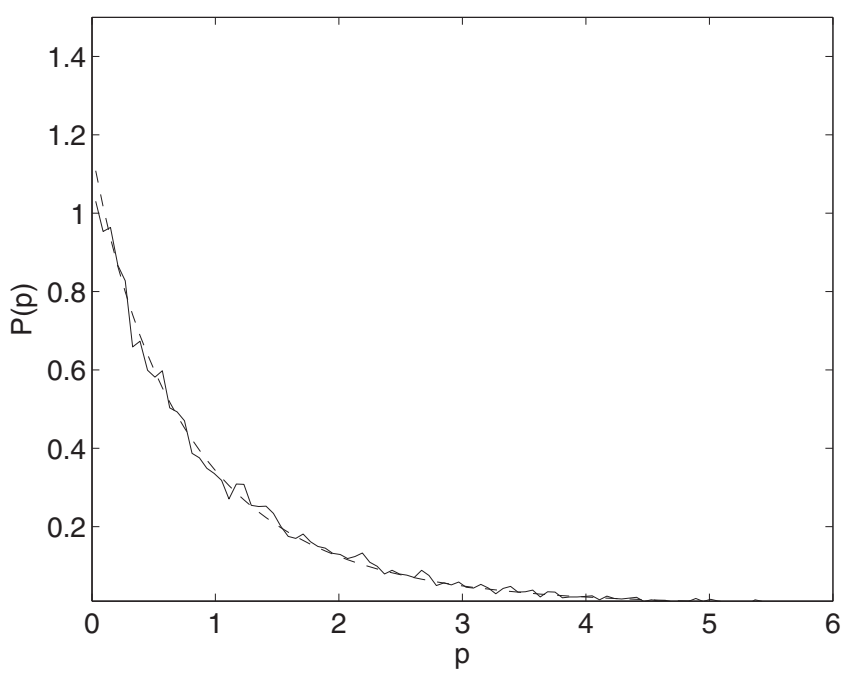

FIG. 7. Same as in the previous figure but for frequencies between 30.85 and $31.5 \mathrm{GHz}$ and $\langle\epsilon\rangle=0.640$.

\section{Current distributions}

The corresponding statistics for the current $\left(j_{\alpha}\right.$ and $\left.j=|\mathbf{j}|\right)$ flowing within the cavity follow the distributions [37]

$$
\begin{gathered}
P\left(j_{\alpha}\right)=\frac{1}{\sqrt{2\left\langle j_{\alpha}^{2}\right\rangle}} e^{-\sqrt{2\left|j_{\alpha}\right| /\left\langle j_{\alpha}^{2}\right\rangle}}, \\
P(j)=\frac{4 j}{\left\langle j^{2}\right\rangle} K_{0}\left(2 \frac{j}{\sqrt{\left\langle j^{2}\right\rangle}}\right),
\end{gathered}
$$

where $K$ is the modified Bessel function of the second kind. The theory is valid for isotropic flow, i.e., the current is not biased towards any direction. This is a reasonable assumption as long as the net flow is weak [39]. Consequently the distributions for the current components $j_{x}$ and $j_{y}$ are also isotropic.

\section{Numerical distributions for currents}

Figures 8 and 9 show numerical distributions of the different current components, $j_{x}, j_{y}$, and $j=\sqrt{j_{x}^{2}+j_{y}^{2}}$. As above, only a section of the cavity has been sampled. There is a clear difference between these distributions; the low-frequency case shows poor agreement with the random wave theory above, while at higher frequencies there is a clear isotropic behavior and the distribution for the total current is much improved. As above, the frequency is thus the most important factor to obtain good agreement with the random wave theory. The small shift in $P\left(j_{y}\right)$ is an effect of the net current within the finite sampled region, an effect that seems negligible at least in the high-frequency region.

\section{E. Pauli stress tensor distributions}

As for the irregular cases above, the stress tensor components $T_{x x}, T_{y y}$, and $T_{x y}$ have been shown to follow specific
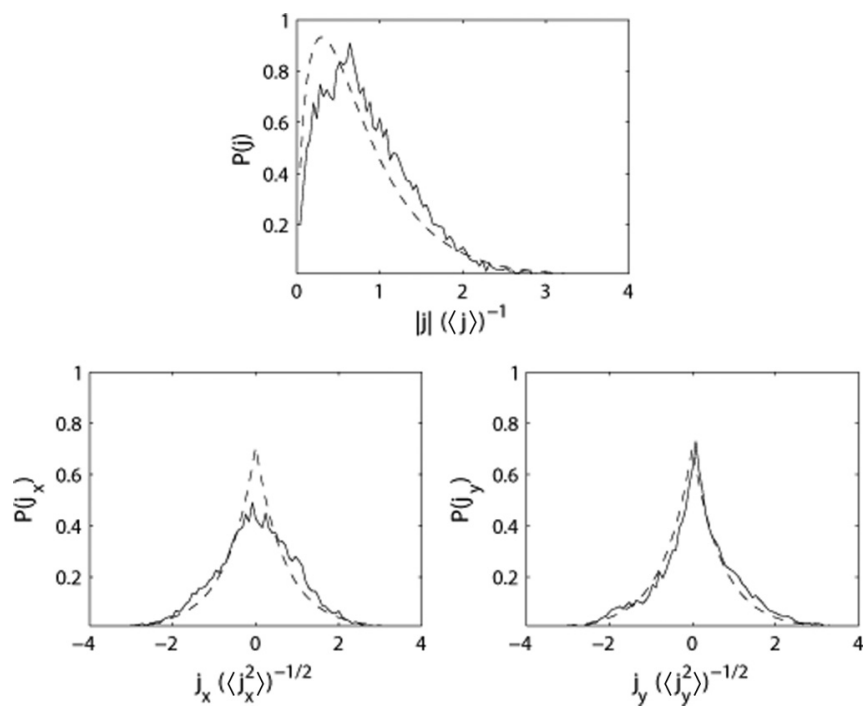

FIG. 8. The numerical distributions (solid line) obtained as an average over 11 wave functions between 4.80 and $5.92 \mathrm{GHz}$ with $\left\langle j_{y}\right\rangle /\langle j\rangle=0.149$. Top: The distribution of $j /\langle j\rangle$. Bottom left and right: The distributions of $j_{x} / \sqrt{\left\langle j_{x}^{2}\right\rangle}$ and $j_{y} / \sqrt{\left\langle j_{y}^{2}\right\rangle}$, respectively. Theoretical distributions (20) and (21) are shown as dashed curves. 
generic distributions [30]. As before, the distribution of these quantities has been derived using the approach of random fields as above. As for the current distributions, the theoretical distributions of the stress tensor components are assumed to be isotropic, i.e., there is no preferred direction. Thus $T_{x x}$ and $T_{y y}$ obey identical distributions. The explicit expression for the different components follows from
Ref. [30],

$$
P\left(T_{\alpha \beta}\right)=\frac{1}{2 \pi} \int_{-\infty}^{\infty} \theta_{\alpha \beta}(a) e^{-i a T_{\alpha \beta}} d a,
$$

where $\theta_{\alpha \beta}$ is the characteristic function for components $\alpha \beta$. Because of symmetry, only two different $\theta_{\alpha \beta}$ are needed, one for $T_{x x}\left(\theta_{x x}\right)$ and one for $T_{x y}\left(\theta_{x y}\right)$. The explicit expressions are

$$
\begin{gathered}
\theta_{x x}(a)=\frac{8}{\sqrt{(1-i a)\left(1-i \epsilon^{2} a\right)[a-i(\sqrt{24}+4)]\left[\epsilon^{2} a-i(\sqrt{24}+4)\right]}} \times \frac{1}{\sqrt{[a+i(\sqrt{24}-4)]\left[\epsilon^{2} a+i(\sqrt{24}-4)\right]}}, \\
\theta_{x y}(a)=\frac{2}{\sqrt{\left[2+\left(\frac{a}{2}\right)^{2}\right]\left[2+\left(\frac{\epsilon^{2} a}{2}\right)^{2}\right]\left[1+\left(\frac{a}{2}\right)^{2}\right]\left[1+\left(\frac{\epsilon^{2} a}{2}\right)^{2}\right]}} .
\end{gathered}
$$

\section{F. Numerical stress tensor distributions}

Averaged stress tensor distributions for low and high frequencies are displayed in Figs. 10 and 11. As in the previous section there is poor agreement with the Gaussian random wave theory at lower frequencies while the fit is quite satisfactory in the other case. As before, the frequency plays a crucial role for agreement with predictions from random wave theory. There is also good agreement with the simulations of quantum transport in an open Sinai billiard with leads explicitly included [30]. Apparently there are no effects of a finite net current as suggested by experiments [30]. The reason is that the frequency is much higher in the present case, which allows for an improved spatial resolution. The asymmetric velocity distribution in Ref. [30] thus turns symmetric at higher frequencies.
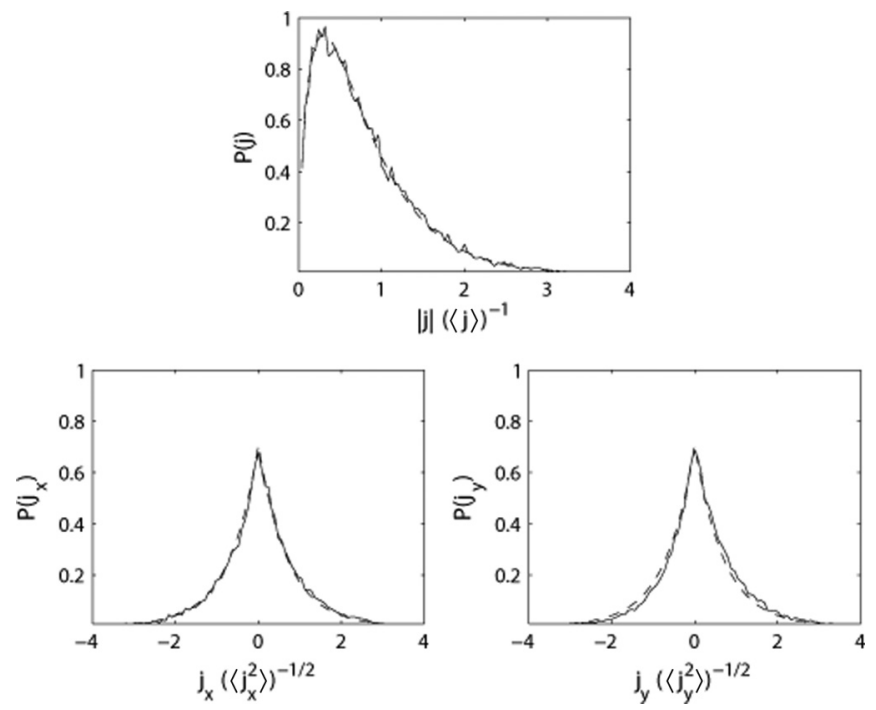

FIG. 9. The numerical distributions (solid line) obtained as an average over 11 wave functions between 30.85 and $31.15 \mathrm{GHz}$ with $\left\langle j_{y}\right\rangle /\langle j\rangle=0.143$. Top: The distribution of $j /\langle j\rangle$. Bottom left and right: The distributions of $j_{x} / \sqrt{\left\langle j_{x}^{2}\right\rangle}$ and $j_{y} / \sqrt{\left\langle j_{y}^{2}\right\rangle}$, respectively. Theoretical distributions (20) and (21) are shown as dashed curves.

\section{SUMMARY AND CONCLUDING REMARKS}

We have explored further a basic model for wave transport in open ballistic cavities such as quantum billiards, microwave resonators, etc., and put it into the general context of $\mathcal{P} \mathcal{T}$ invariance. The model makes use of imaginary potentials which mimic, for example, connects to the source and drain of a quantum dot. The model was applied to a planar quantum or microwave cavity in Ref. [7]. Wave function morphology and flow patterns for low-energy or low-frequency modes were found to be in good agreement with experiments.

The purpose of the present paper was to probe the model further by extending the previous studies to high frequencies at which chaotic dynamics is to be expected. The complex $\mathcal{P} \mathcal{T}$-invariant solutions of a Schrödinger or Helmholtz equation with imaginary potentials were analyzed in terms of the universal HQM statistical distributions for wave chaotic
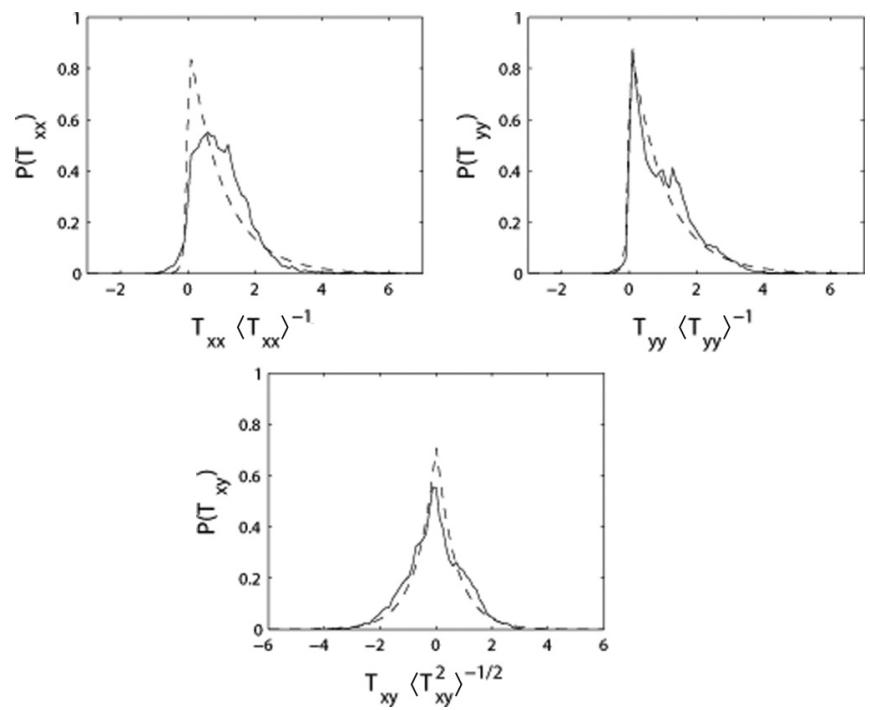

FIG. 10. The numerical distributions for the different stress tensor components (solid line) obtained as an average over 11 wave functions with frequency between 4.80 and $5.92 \mathrm{GHz}$. Top left and right: The distributions of $T_{x x}$ and $T_{y y}$, respectively. Bottom left: The distribution of $T_{x y}$. Predictions from the Gaussian random wave theory (22)-(24) are shown as dashed curves. 

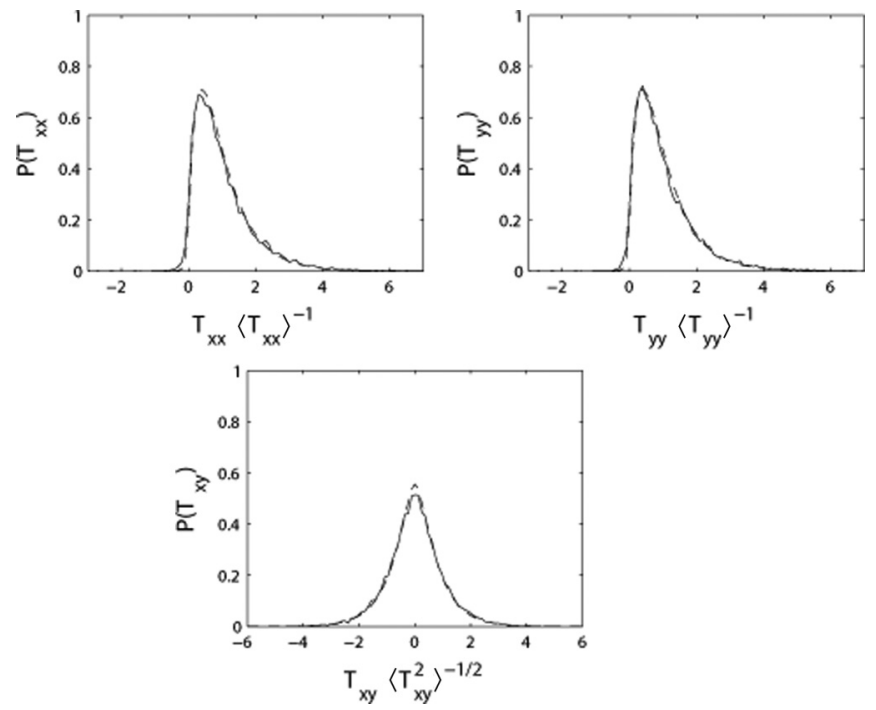

FIG. 11. The numerical distribution of the different stress tensor components (solid line) obtained as an average over 11 wave functions with frequency between 30.85 and $31.15 \mathrm{GHz}$. The distributions of $T_{x x}$ and $T_{y y}$, respectively. Bottom left: The distribution of $T_{x y}$. Predictions from the Gaussian random wave theory (22)-(24) are shown as dashed curves.

systems, in this case, the conventional random wave statistical distributions for densities, currents, and the Pauli stress tensor components. The agreement between the present approach and the universal distributions from HQM Gaussian random wave theory is quite satisfactory provided that $\mathcal{P} \mathcal{T}$ symmetry is obeyed. (This point is important. If there would be a $\mathcal{P} \mathcal{T}$ breaking, there would obviously be a breakdown of particle conservation, etc.) In particular, the above good results for the stress tensor components are most encouraging since they probe higher derivatives, i.e., we get a more stringent test of the model. We have also found good qualitative agreement with experiments [30], but note that the experimental regime needs to be extended to higher frequencies and smaller mesh for improved resolution. This point is of principle interest as the random wave Gaussian distributions derive from HQM rather than NHQM.

In summary, we conclude that our basic non-Hermitian model with imaginary potentials for the source and drain works well for nominally $\mathcal{P} \mathcal{T}$-symmetric ballistic quantum dots or cavities. It is relatively easy to apply from a computational point of view as the more cumbersome external leads may be avoided. We have also found that simple perturbation theory may be quite useful for soft imaginary potentials. It may be employed, for example, for "quick scanning and optimization" of the impact of different positions of (multi)leads to guide, for example, the design of more complex devices and also for networks as outlined in Ref. [25] and possibly filtering. These aspects should be of great practical value when dealing with transport in open 3D objects such as cubes, terahedra, ellipsoids, cylindrical rods, etc., and ensembles of objects such as these. In such cases finite difference computations tend to be overly demanding in terms of time and memory. Finally, $\mathcal{P} \mathcal{T}$-symmetry breaking and phase rotations associated with complex level repulsions invite further studies, both theoretical and experimental, including also dimensions higher than two.

\section{ACKNOWLEDGMENT}

We wish to thank Professor Jon Bird, University at Buffalo, The State University of New York, for helpful comments and discussion.
[1] D. K. Ferry, S. M. Goodnick, and J. P. Bird, Transport in Nanostructures, 2nd ed. (Cambridge University Press, Cambridge, U.K., 2010).

[2] H.-J. Stöckmann, Quantum Chaos: An Introduction (Cambridge University Press, Cambridge, U.K., 1999).

[3] F. J. Fahy, Sound Intensity, 2nd ed. (E \& FN Spoon, London, 1995).

[4] C. C. Chen, C. C. Liu, K. W. Su, T. H. Lu, Y. F. Chen, and K. F. Huang, Phys. Rev. E 75, 046202 (2007).

[5] O. Bengtsson, J. Larsson, and K.-F. Berggren, Phys. Rev. E 71, 056206 (2005)

[6] For a recent overview on microwave billiards, see, for example, U. Kuhl, Eur. Phys. J.: Spec. Top. 145, 103 (2007).

[7] K.-F. Berggren, I. I. Yakimenko, and J. Hakanen, New J. Phys. 12, 073005 (2010).

[8] K.-F. Berggren and I. I. Yakimenko, J. Sib. Fed. Univ. Math. Phys. 3, 280 (2010).

[9] H. Feshbach, C. E. Porter, and V. F. Weisskopf, Phys. Rev. 96, 448 (1954).

[10] L. I. Schiff, Quantum Mechanics (McGraw-Hill, New York, 1968).

[11] J. P. Bird, R. Akis, D. K. Ferry, A. P. S. de Moura, Y.-C. Lai, and K. M. Indlekofer, Rep. Prog. Phys. 66, 583 (2003).
[12] M. Barth and H.-J. Stöckmann, Phys. Rev. E 65, 066208 (2002).

[13] W. Pauli, in Quantentheorie, Handbuch der Physik Vol. XXIV (Springer, Berlin, 1933).

[14] W. Pauli, General Principles of Wave Mechanics, English translation by P. Achuthan and K. Ventkaesan (Springer, Berlin, 1980).

[15] R. E. Wyatt, Quantum Dynamics with Trajectories, Introduction to Quantum Hydrodynamics, Springer Series in Interdisciplinary Applied Mathematics (Springer, New York, 2005), Vol. 28.

[16] C. M. Bender and S. Boettcher, Phys. Rev. Lett. 80, 5243 (1998).

[17] C. M. Bender, M. DeKieviet, and S. P. Klevansky, Philos. Trans. R. Soc. A 371, 20120523 (1989).

[18] C. M. Bender, Am. J. Phys. 71, 1095 (2003).

[19] S. Weigert, Czech. J. Phys. 54, 1139 (2004).

[20] J. G. Muga, J. P. Palaob, B. Navarroa, and I. L. Egusquizac, Phys. Rep. 395, 357 (2004).

[21] D. K. Ferry and J. R. Barker, Appl. Phys. Lett. 74, 582 (1999).

[22] D. K. Ferry, R. Akis1, A. M. Burke1, I. Knezevic, R. Brunner, R. Meisels, F. Kuchar, and J. P. Bird, Fortschr. Phys. 61, 291 (2013). 
[23] T. Lundberg, E. Sjöqvist, and K.-F. Berggren, J. Phys.: Condens. Matter 10, 5583 (1998).

[24] K. Varga and S. T. Pantelides, Phys. Rev. Lett. 98, 076804 (2007).

[25] B. D. Wibking and K. Varga, Phys. Lett. A 376, 365 (2012).

[26] M. Znojil, Phys. Rev. D 80, 045009 (2009).

[27] C. E. Rüter, K. G. Makris, R. El-Ganainy, D. N. Christodoulides, M. Segev, and D. Kip, Nat. Phys. 6, 192 (2010).

[28] A. Guo, G. J. Salamo, D. Duchesne, R. Morandotti, M. VolatierRavat, V. Aimez, G. A. Siviloglou, and D. N. Christodoulides, Phys. Rev. Lett. 103, 093902 (2009).

[29] Y. N. Joglekar, C. Thompson, D. D. Scott, and G. Vemuri, Eur. Phys. J. Appl. Phys. 63, 30001 (2013).

[30] K.-F. Berggren, D. N. Maksimov, A. F. Sadreev, R. Höhmann, U. Kuhl, and H. J. Stöckmann, Phys. Rev. E 77, 066209 (2008); 79, 019901(E) (2009).

[31] J. S. M. Anderson, P. W. Ayers, and J. I. Rodriguez Hernandez, J. Phys. Chem. A 114, 8884 (2010).
[32] A. Iserles, A First Course in the Numerical Analysis of Differential Equations (Cambridge University Press, Cambridge, U.K., 2003).

[33] A. Richter, Phys. Scr., T 90, 22 (2001).

[34] For a topical review on non-Hermitian Hamilton operators and the physics of open quantum systems, see I. Rotter, J. Phys. A 42, 153001 (2009).

[35] Quantum Physics with non-Hermitian Operators: Theory and Experiment, edited by J. Bird, R. Kaiser, I. Rotter, and G. Wunner, special issue of Fortschr. Phys. 61 (2-3) (2013).

[36] S. Bittner, B. Dietz, H. L. Harney, M. Miski-Oglu, A. Richter, and F. Schäfer, Phys. Rev. E 89, 032909 (2014).

[37] A. I. Saichev, A. F. Sadreev, and K.-F. Berggren, J. Phys. A: Math. Gen. 35, L87 (2002).

[38] H. Ishio, A. I. Saichev, A. F. Sadreev, and K.-F. Berggren, Phys. Rev. E 64, 056208 (2001).

[39] A. F. Sadreev and K.-F. Berggren, Phys. Rev. E 70, 026201 (2004). 